Article

\title{
Human Cognition, Patterning and Deacon's Absentials: The Value of Absent-Mindedness in the Sense of Minding What Is Absent
}

\author{
Marlie Tandoc ${ }^{1}$ and Robert K. Logan ${ }^{2, *}$ (CD \\ 1 Book and Media Studies, University of St. Michael's College, University of Toronto, 60 St. George, \\ Toronto, ON M5S 1A7, Canada; marlie.tandoc@mail.utoronto.ca \\ 2 Department of Physics, University of Toronto, 60 St. George, Toronto, ON M5S 1A7, Canada \\ * Correspondence: logan@physics.utoronto.ca
}

Received: 3 September 2018; Accepted: 18 September 2018; Published: 21 September 2018

\begin{abstract}
Important aspects of human cognition are considered in terms of patterning, which we claim represents a shift from focusing on what is present to what is absent. We make use of Deacon's notion of absentials and apply it to the patterning that underscores human cognition. Several important aspects of human cognition are considered that represent a shift from focusing on what is present to what is absent, namely, language as representing the transition from percept to concept-based thinking, mathematical grouping and patterning of items into sets that gave rise to verbal language, as well as imaginative thinking which is so critical for the development of the arts, mathematics and science. The connection between information and absence is also examined, in which we claim that information is an absential, paralleling an idea of Deacon's.
\end{abstract}

Keywords: absentials; abiotic matter; patterning; constraints; cognition; imagination

There is more here than stuff. There is how this stuff is organized and related to other stuff

-Terrence Deacon

The spoken word was the first technology by which man was able to let go of his environment in order to grasp it in a new way

-Marshall McLuhan

\section{Introduction}

Important aspects of human cognition are considered in terms of patterning, a key factor in the development of human cognition and culture, which also represents a shift from focusing on what is present to what is absent. We make use of Deacon's notion of absentials by which he understands the emergence of life and sentience from abiotic matter in his book Incomplete Nature. Logan and Tandoc asserted in "Thinking in Patterns \& Patterns of Human Thought as Opposed to AI Data Processing" that the patterning that underscores human cognition is re-examined in terms of Deacon's absentials. Several important aspects of human cognition are considered that represent a shift from focusing on what is present to what is absent, namely, language as representing the transition from percept to concept-based thinking, as described in The Extended Mind, mathematical grouping and patterning of items into sets that gave rise to verbal language, as well as imaginative thinking which is so critical for the development of the arts, mathematics and science. The connection between information and absence is also examined, in which we claim that information is an absential, paralleling an idea of Deacon's. 
In ref. [1] (available through open access at http://www.mdpi.com/2078-2489/9/4/83) we posited that a key factor in the development of human cognition and culture was the unique ability of humans to both recognize and create patterns, which we will henceforth refer to as patterning. Patterning led to the emergence of verbal language, the ability to deal with information overload, conceptualization, imagination, invention, mathematics, and the sciences, both natural and social. Patterning is also an essential part of the visual arts, music, and narratives which are oral, literate and cinematic in nature.

In that study [1] we proposed that the ability of humans to identify and create patterns led to the unique aspects of human cognition and culture as a complex emergent dynamic system consisting of the following human traits that co-emerged: patterning, social organization, rudimentary set theory or categorization, spoken language, and the ability to deal with information overload. We argued that these traits are interrelated, as they all involve the ability to flexibly manipulate information from our environments via pattern restructuring. We argued that the human mind is the emergent product of a shift from external percept-based processing to a concept and language-based form of cognition [1] based on patterning. We described the evolution of human cognition and culture, arguing that they emerged from the ability of humans to think in terms of patterns. One example of the way patterning contributed to human cognition is the role it played in the emergence of language, which involved the ability of humans to create patterns by distinguishing a set of objects or activities that are similar, differentiating them from other objects or activities, and giving a name to that set, namely, a word.

\section{Deacon's Incomplete Nature}

In this essay, we will show that while patterning involves relationships among the elements that make up the pattern or the set, the most essential feature that makes the pattern possible is actually absent. What is absent is the definition of what constitutes the pattern or set, which is an absential, as defined by Deacon [2] (p. 2) in his groundbreaking study Incomplete Nature. According to Deacon, life and sentience emerged from abiotic matter in terms of absentials defined as "there is something not-there there." The something that is "not-there there" is the way the abiotic matter is organized, or as Deacon proposed explaining how life and sentience emerges from abiotic matter or "stuff": "There is more here than stuff. "There" is how this stuff is organized and related to other stuff [2] (p. 544)." This according to Deacon is how life and sentience emerges from abiotic matter in terms of absentials or "something not-there". Those who are unfamiliar with Deacon's work might have a look at a review and precís of his book [3].

The thesis that we develop in this article is dependent on the ideas that Deacon developed in his book Incomplete Nature. Deacon developed the paradoxical notion that it is something absent, an absential, that allows him to understand the origin of life and sentience. Deacon [2] (p. 2) contends that one cannot relate the phenomena of "a function, reference, purpose, or value", so essential for understanding life and cognition to physical matter, and that each of these phenomena "is in some way incomplete." Deacon suggests that organization be thought of in terms of constraints that create absences, or what is not there. Constraints prevent things from happening, and hence, create absenses.

But although the specific absences that constitute a constraint do not suffer the epiphenomenality of descriptive notions of organization, they are nevertheless explicitly not anything that is present. This requires that we show how what is absent is responsible for the causal power of organization and the asymmetric dynamics of physical or living process [2] (p. 195)

Deacon associates information with constraints, making reference to Kauffman, Logan, et al. [4], where the notion that information and constraints are intimately connected was developed. 


\section{Patterning, the Basis of Human Cognition Involves Something That Is Absent}

We suggest that, in addition to understanding the nature of life and sentience in terms of absentials or something that is not there a la Deacon, the patterns in the arts and sciences created by human sentience or cognition can also be understood in terms of something that is not there. This is consistent with the fact that living organisms and sentience are themselves essentially patterns. Furthermore, human cognition not only recognizes and identifies patterns, but it also creates patterns in the form of language, mathematics, science, music, the visual arts, and narratives to mention only a few. Indeed, we agree with Deacon that there is immense value in thinking in terms of absences not only for the natural sciences, but also for human cognition and culture in general. For example, Deacon concurs that there are some "serious inadequacies" with our current scientific theories, as well our notions of life in general, that thinking in terms of absences could remedy:

A counterintuitive figure/background reversal, focusing on what is absent rather than present, offers a means to repair some of the serious inadequacies of our conceptions of matter, order, life, work, information, representation and even consciousness and conceptions of value [2] (p. 44)

We believe that the human mind already excels at this figure-ground reversal, but in an intuitive, everyday sense, which allows us to "repair" our personal understandings of our world. Focusing via patterning on what is absent rather than present is the property of the human mind that allows us to extract information and make meaning of our world. This paper aims to focus on some of the ways in which human cognition has already evolved to think in terms of absences, via recognizing and minding the organizational properties of our world. We will examine several of these patterning processes of the human cognition including language, mathematics, science, and imaginary thinking, as well as the cultural concepts or artifacts that such thinking creates. The issue of formalizing this type of thinking in the natural sciences, as noted by Deacon, will also be discussed.

We will first review how ref. [1] came to the conclusion that patterning is an essential and unique feature of human cognition and culture. We will then describe Deacon's notion of absentials and show how patterning also involves absentials, or how patterns involve something that is not there in the elements making up the pattern itself. To paraphrase Deacon, there is more to the pattern than the elements making up the pattern, there is the way in which these elements are organized, and how they relate to each other.

\section{Thinking in Patterns and the Patterns of Human Thought}

As humans gained complete control of fire and began to live around their campfires in clans instead of nuclear families, information overload developed. Logan [5] argued that verbal language emerged to deal with this information overload. Verbal language, which replaced the mimetic communication of pre-verbal vocalization, gestures, hand signals, and body language, allowed for conceptualization, and hence, planning and the co-ordination of group activity. The first words acted as concepts that represented all of the percepts that were associated with that concept. The use of the word "water", for example, instantly triggers all of one's direct experiences and perceptions of water, such as the water we drink, the water we cook with, the water we wash with, rain water, and the water that is found in rivers, ponds, lakes, and oceans. The word or concept of water is not there; only the actual material water made up of $\mathrm{H}_{2} \mathrm{O}$ molecules is there.

Before humans had verbal language, the brain was basically a percept processor; however, with language, the brain became a mind that was capable of conceptualization, with the ability to plan and co-ordinate its activities. The mind is the brain plus language (mind = brain + language). Language is not just a medium to communicate thoughts; it is also the medium in which thoughts are formulated. Indeed, the shift from percept-based to concept-based thinking that emerges with language parallels Deacon's emphasis on a shift from solely what is present to what is absent as well. The words of a language that are absent in the physical world describe the objects of the world that are present in the 
world in the physical sense. This parallels McLuhan's idea that language was the first tool we used to "let go of [our] environment in order to grasp it in a new way," i.e., to let go of our environment perceptually and grasp it conceptually. Percepts are present in the material world, in the sense they are sensations detected by the subject; in contrast, concepts do not have a material presence, as they are thoughts in the mind of the subject. We are quite comfortable with the idea that a picture may be worth a thousand words. However, in our view, a word also brings to mind thousands of individual images (percepts). It is in this absence of any present perceptual experience that the power of language and conceptual thinking resides.

Language gave us the tool to formulate "absences" in terms of concepts or words, and move from a percept-based form of thinking to a concept-based form of cognition which is capable of dealing with things not present in the here and now. For example, Alison Gopnik [6] argued in her book Philosophical Baby that a major part of childhood cognition is imagination, or the ability to consider "what could have been" and "what could be." Concepts are imagined, not perceived or sensed. It takes imagination to conceive. For example, it takes imagination to see that the various forms of water that we encounter perceptually are related. One must imagine what is not there, namely, the categories that language allows us to create.

Forming or positing a pattern is a form of hypothesizing. A hypothesis is something that is not there; there is only the data that is sensed or perceived from which the hypothesis is formulated or imagined. A paradox: Hypothesizing is a form of minding what is absent, which is the opposite of being absent-minded. Similarly, creative impasses are also highly reflective of Deacon's argument that we have stubbornly fixated too long on the "stuff" in the world. Creative insight may be defined as the ability to reorganize knowledge (i.e., patterning) to overcome a particular problem. This is why, when faced with a problem, it is often recommended that we "sleep on it" or "take a break and come back to it". When we return to the problem, the "stuff", of course, is exactly the same as it was before; nothing has changed in the physical material world. But perhaps we may recognize a new organizational property in-between the "stuff". Creative thought and creating hypotheses is the perfect example of thinking in terms of absences. Thinking in absences frees us from the material world, and gives us a mental sandbox of possibilities and hypotheses to play with. Indeed, just like manipulating various parameters or constraints in a mathematical model or computer simulation to produce different outcomes, imagination is also like playing around with constraints in our mental sandbox to create novel scenarios, of what could have been, what could be, or what will be. Thus, viewing imagination as the importance of "absence" in Deacon's notion of constraints and absentials demonstrates that a lot of human cognition parallels the figure-ground reversal, as first formulated by Marshall McLuhan, and later incorporated in Deacon's shift from what is present to what is absent.

The term constraint thus denotes the property of being restricted or being less variable than possible, all other things being equal, and irrespective of why it is restricted [2] (p. 193)

\section{Patterning and the Origin of Language}

A key cognitive ability that led to language was to see the pattern in all of one's experiences or percepts with water, and to use the word "water" accordingly, acting as a concept to describe the set of all of one's experiences or percepts of water. We argue that the human mind is the emergent product of a shift from external percept-based processing to a concept and language-based form of cognition based on patterning. Logan and Pruska-Oldenhof [7], in a book entitled A Topology of Mind —Spiral Thought Patterns, the Hyperlinking of Text, Ideas and More, developed the thesis that "the human mind is intrinsically verbal and mathematical, and that language and mathematical thinking in the form of set theory co-emerged at the dawn of the emergence of Homo sapiens." A word is created as a concept representing the set of percepts that relate to that concept.

The unique human ability of patterning, i.e., the ability to identify and create patterns, led to the flowering of human cognition and culture based on conceptualization. We claim therefore that humans basically think in terms of patterns. We will show that patterning and patterns themselves, which 
are so essential for the emergence of verbal language and all that followed in its wake, are based on something that is missing, or an absential, as defined by Deacon [2]. We therefore turn to a description of absentials and the role they play in the emergence of life and sentience from abiotic matter.

\section{Deacon's Incomplete Nature and the Role of Absentials in the Emergence of Life and Sentience}

Deacon [2] in Incomplete Nature: How Mind Emerged from Matter attempts to understand how life, sentience, and the human mind emerged from abiotic matter. He posits that these phenomena are not the result of the properties of the matter that give rise to them, but rather, the way that the abiotic physical matter is organized. The pattern of the organization of this abiotic matter that gives rise to life and sentience is what he defines as an absential. The matter is there physically, but the pattern is an absential that is not explicitly there physically.

Deacon contends that one cannot explain the phenomena of "function, reference, purpose, or value" in terms of the properties of physical matter, and that each of these phenomena that are essential to life and sentience "is in some way incomplete [2] (p. 2)". Each of these phenomena cannot be explained in physical terms or linked to physical processes, which is why Deacon suggests that there is something missing which he labels as an "absential" or an "absential feature [2] (p. 3)", his neologism for what is missing from our understanding of the physical dimension of these phenomena. Absential features are the "defining property of life and mind [2] (p. 3)."

Deacon's formulation of absentials represents what McLuhan would call a figure/ground reversal, in which, instead of focusing on matter, one focuses on their interactions, or as Deacon puts it:

A counterintuitive figure/background reversal, focusing on what is absent rather than present, offers a means to repair some of the serious inadequacies of our conceptions of matter, order, life, work, information, representation and even consciousness and conceptions of value .... It requires reframing the way we think about the physical world in thoroughly dynamical, that is to say, process terms, and recasting our notion of causality in terms of something like the geometry of this dynamics, instead of thinking in terms of material objects in motion affected by contact and fields of force. [2] (p. 44)

Deacon suggests that organization be thought of in terms of constraints, or what is not there, i.e., absences. It is the constraints that give rise to the pattern.

Constraint is a property of a collection or ensemble of some sort, but a negative property. It is a way of referring to what is not exhibited, but could have been, at least under some circumstances ... But although the specific absences that constitute a constraint do not suffer the epiphenomenality of descriptive notions of organization, they are nevertheless explicitly not anything that is present. This requires that we show how what is absent is responsible for the causal power of organization and the asymmetric dynamics of physical or living process [2] (pp. 193, 195)

\section{Absentials and Patterning}

We suggest that patterning or the creation and identification of patterns, which is so essential to the origin of verbal language and conceptual thought in human cognition that verbal language made possible, also depend on absentials. The absential of a pattern of elements is the something that it is missing. It is the constraint that allows the elements making up the pattern to be recognized as a pattern, but it, itself, is not part of the pattern. A set is, in a certain sense, also a pattern. The set of all fruits that are apples is composed only of apples, yet the constraint or definition of what is considered to be an apple is not part of the set; it is absent. The set of all even numbers does not contain the constraint that for a number to be a member of the set of even numbers, its division by two must be a whole number. This defining constraint for the set of even numbers is not a member of the set itself. The constraint or information that defines a set is not part of the set, and hence, is absent, i.e., it is 
an absential. In other words, the qualia, or the whatness that defines a set, a category, or a pattern is an absential.

We will claim that basically, the absential, which Deacon defines as a constraint, is information based on ideas developed in [4]. In Incomplete Nature, Deacon begins Chapter 13, entitled Significance, with the following super heading:

The first surprise is that it takes constraints on the release of energy to perform work, but it takes work to create constraints. The second surprise is that constraints are information and information is constraint-Stuart Kauffman, Personal Communication.

This Personal Communication references the article "Propagating Organization: An Enquiry [4]". In this referenced article, the following claim is made:

Constraints are information and information is constraints. The first part of this twosome, constraints are information is, we believe, secure. The second part, information is constraints, may be more problematic [4].

The idea that constraints are the information, instructional, or biotic information, was first formulated in a conversation when Stuart Kauffman asked Robert Logan how cells were able to do the work to build constraints that allowed them to convert external energy from their environment into work if the constraints did not exist in the first place (a chicken and egg problem). Logan told Kauffman he could not answer his question but that the constraints are the information, which he came up with by channeling Marshall McLuhan and his notion that "the medium is the message".

If the absential that defines a pattern is a constraint, and a constraint is information as is claimed in [6], then we feel justified in claiming that information is an absential. In fact, Deacon makes the same claim that, "Information is the archetypical absential concept [2] (p. 374)." The information about or the organization of a system, whether it is organic or abiotic, is an absential. The natural sciences are forms of information that are absentials, in that they are not part of the phenoemona they are describing. The phenomena that natural science describes is present in the material sense, but the description is not present materially, and hence, is absent.

Another argument that information is absential is as follows: Constraints which are information create absences. How? Consider a glass of water. The glass is a constraint preventing the water from leaving the glass, and hence, creates an absence of the water outside of the glass. Names and words are also absentials-words are concepts, and therefore absentials. Absent are all the percepts not related to the word or the concept the word represents.

If constraints are absentials and also information, then perhaps it follows that information is also an absential. Information is grammatically a noun, but it describes a process of informing and informing is a verb. As such, information appears even further removed or absent in the material sense. Information is not a currency, a material thing, an object, or as [2] (p. 333) puts it, it is not "a physical commodity; a kind of stuff that one can acquire, store, sell, move, lose, share, and so on"; instead, information is a process. It is a word that describes a process, i.e., how parts interconnect, relate, and work together in a system.

Let's consider whether the information that a stop sign conveys is an absential? The stop sign is only the red octangle with the letters $\mathrm{s}, \mathrm{t}, \mathrm{o}$, and $\mathrm{p}$ on it, and therefore, the information is not there in the material sense; it is absent. The red octangle and the letters: $s, t, o$, and $p$ are not the information. The information is in the process of the interpretation of the stop sign by the motorist. It is the effect on the motorist.

If information is a process, then measuring it should be, in terms of its effects, not the number of its bits, as Shannon [8] defined it. If information, on the other hand, is a noun, a material thing, then yes, the number of bits is a measure of it. But information is not a static thing, but rather, a dynamic process, and therefore, not a material thing that can be measured in terms of the number of bits of which it is composed. Although "information" is a noun, it certainly behaves like a verb. Linguistically 
and grammatically, the word "information" is a noun, but in actuality it is a process, and hence, is verb-like. The word is derived from Latin through French by combining the word "inform", meaning giving a form to the mind, with the ending "ation", denoting a noun of action. This earliest definition refers to an item of training or molding of the mind. Shannon's form of information is a noun or a thing. Both MacKay's [9] "information is a distinction that makes a difference" and Bateson's [10] (p. 428) "information is a difference that makes a difference" are definitions that assume information is a verb or a process. These are two different ways in which the same term, information, is used; one is noun-like, in the case of Shannon, while the other is verb-like, in the case of MacKay and Bateson. Basically, what we are suggesting is that Shannon's theory is not so much a theory of information, but a theory about the transmission of signals, since it does not deal with the interpretation of the signal by the receiver, which is an essential element of information.

The connection of information with the idea of giving form to the mind is the key to the notion that information is not a thing, but rather, a process by which the mind is formed or informed, and hence, it is intimately tied to the meaning and the effect on the recipient of the information. What we normally call information as a thing in our everyday use of the term is actually information as signs, which only inform when they have meaning for their recipient or receiver, the interpreter of the signs.

\section{Culture as an Absential: The Product of the Patterns of Human Thought}

As the human mind has often been described as an information-processor, it makes sense that our cognitive abilities and cultural concepts have evolved to best process information, a Deacon absential. Culture can be viewed as the product of the transmission of information, with the human mind acting as the medium for that information. Hence, a la McLuhan [7,11], "the medium is [indeed] the message".

Because humans think and process concepts in terms of absentials, our cultural concepts are also absential as well. For example, in a recent article in the journal of Theology, Moritz [12] argued that the notion of God (perhaps one of the most ubiquitous aspects of human culture) is an example of Deacon's absential concept. As the notion of God is prevalent across human cultures all over the world, it does seem that there is a property of the human mind, a "way in which we think, that allowed this idea to emerge across space and time. We believe this "way" to be thinking in terms of absences and patterns. Indeed, owing to the fact that there are universal concepts such as God, religion, honor, music, art, science, mathematics, marriage, etc. across diverse cultures, as Brown [13] has suggested, it follows that certain properties of human cognition, such as thinking in absences and patterns, over time, converged on these abstract absential concepts. In a similar way, Deacon's notion of teleodynamics, of self-reproducing self-correcting systems, has also led to the universal human phenomena of culture, language, organization, science, economics, and technology [3]. As teleodynamics requires that "presence and absence must be mutually inter-defined" Ref. [14], it can also be argued that these human cultural concepts are also largely defined by their absential qualities. Cultural artifacts are formulated in terms of absentials produced by the human mind, which is capable of thinking in terms of language and concepts, both of which are, in turn, absentials.

Author Contributions: Both authors contributed to this article equally.

Funding: There is no external funding.

Conflicts of Interest: The authors declare no conflict of interest.

\section{References}

1. Logan, R.K.; Marlie, T. Thinking in Patterns \& Patterns of Human Thought as Opposed to AI Data Processing. Information 2018, 9, 83. [CrossRef]

2. Deacon, T.W. Incomplete Nature; WW Norton \& Company: New York, NY, USA, 2011.

3. Logan, R.K. Review and Précis of Deacon's Incomplete Nature: How Mind Emerged from Matter. Information 2012, 6, 290-306. [CrossRef] 
4. Kauffman, S.; Logan, R.K.; Este, R.; Goebel, R.; Hobill, D.; Smulevich, I. Propagating Organization: An Inquiry. Biol. Phil. 2007, 23, 27-45. [CrossRef]

5. Logan, R.K. The Extended Mind: The Emergence of Language, the Human Mind and Culture; University of Toronto Press: Toronto, ON, Canada, 2007.

6. Gopnik, A. The Philosophical Baby; Picador: London, UK, 2010.

7. Logan, R.K.; Pruska-Oldenhof, I. A Topology of Mind-Spiral Thought Patterns, the Hyperlinking of Text, Ideas and More; Springer: Cham, Switzerland, 2018.

8. Shannon, C.E. A Mathematical Theory of Communication. Bell Syst. Tech. J. 1948, 27, 379-423, $623-656$. [CrossRef]

9. MacKay, D. Information, Mechanism and Meaning; MIT Press: Cambridge, MA, USA, 1969.

10. Bateson, G. Steps to an Ecology of Mind; Paladin, Frogmore: St. Albans, UK, 1973.

11. McLuhan, M. Understanding Media: Extensions of Man; Mac Graw Hill: New York, NY, USA, 1964.

12. Moritz, J.M. How Absence Makes Things Grow: Absential Metaphysics and the Teleodynamics of God as Love. Theol. Sci. 2016, 14, 449-455. [CrossRef]

13. Brown, D.E. Human Universals; Mac Graw Hill: New York, NY, USA, 1991.

14. Deacon, T.W.; Cashman, T. Steps to a Metaphysics of Incompleteness. Theol. Sci. 2016, 14, 401-429. [CrossRef]

(C) 2018 by the authors. Licensee MDPI, Basel, Switzerland. This article is an open access article distributed under the terms and conditions of the Creative Commons Attribution (CC BY) license (http:/ / creativecommons.org/licenses/by/4.0/). 